\title{
Information Environment Metadata Schema Registry
}

\author{
Emma Tonkin and Alexey Strelnikov \\ UKOLN, University of Bath, UK, BA27AY \\ e.tonkin@ukoln.ac.uk, \\ a.strelnikov@ukoln.ac.uk
}

\begin{abstract}
Several metadata schemas focusing on Dublin Core metadata are now in operation around the world. In this demonstration, we support participants in exploring various components that make up the IEMSR metadata schema registry: a desktop tool enabling creation of simple DC vocabularies and application profiles and their addition to the registry; a Web client that enables the registry to be browsed and searched; a series of prototype tools that demonstrate the use of the machine-to-machine SPARQL endpoint for practical scenarios such as internationalization and complex application profile creation using an explicitly stated entity-relationship model.
\end{abstract}

Keywords: Metadata schema registry, application profile, Dublin Core.

\section{Introduction to the IEMSR}

The metadata schema registry is a service or application that collects together information from a variety of sources on the subject of metadata schemas, structures, terms/elements, and potentially terminologies and value encodings. Schema registries are designed and used for a variety of purposes, such as promotion of the reuse of existing vocabulary, standardisation, and discovery of appropriate terms. Humanreadable descriptions of vocabulary terms and of their uses in given contexts may be provided alongside machine-readable mappings of equivalency, term hierarchy, etc. Metadata schemas and vocabularies represent work that should be retained, published and curated responsibly; low availability or high barrier to access of existing metadata structures and vocabularies discourages reuse. Johnston[1] identifies a number of functions common to metadata schema registries: disclosure/discovery of information about metadata terms and relationships between metadata terms; mapping/inferencing services connecting terms; verification of the provenance or status of metadata terms; discovery of related resources (aggregations, usage guidelines, bindings etc). Several metadata schema registries focusing on Dublin Core metadata exist, including the NSDL metadata registry[4] and the IEMSR metadata schema registry, a prototype service currently under evaluation at UKOLN[3].

This session is intended to focus primarily on the IEMSR, which consists of several tools: a SPARQL endpoint for data access; a web client; a desktop client; a set of design/prototyping tools. This demonstration session will examine these in detail, ideally via example scenarios provided by participants (standard examples will also 
be available); for example, development of a sample AP, or exploration of a structured AP. Participants may wish to bring along some documentation, such as an informal set of requirements, describing a scenario they wish to explore. The target audience of this demonstration includes metadata schema, application profile and vocabulary developers; those with an interest in Semantic Web technologies; those making practical use of metadata, particularly DC, such as registry managers and developers; developers interested in making use of information about metadata standards as part of software development in general terms; for example, those with an interest in web engineering; those with a more general interest in metadata standards, including those who do not closely follow developments in the DCMI world. Previous demonstrations have taken place in a light-hearted 'bar camp' atmosphere; participants are encouraged to engage with the concept on their own terms. Because the IEMSR is a network-based application, network access will be required throughout the demonstration. It is possible for the IEMSR to be installed locally or booted from a live CD or USB drive, but access to the primary IEMSR endpoint and websites may be preferred, as this enables access to online documentation and tutorial information.

\section{Conclusion}

The IEMSR, a pilot service currently in a review phase, continues to develop and change with the changing needs of the environment within which it operates. Potential applications of a metadata registry have themselves evolved greatly in the past few years, and as the amount of available data has increased and expectations on metadata as a technologyhave shifted. Ongoing work in development of application profiles for deployment within national and international repository ecosystems has highlighted the question of appropriate development/evaluation methodologies; the schema registry is viewed as a resource able to support rapid prototyping and toolsets developed for this purpose. We aim to provide participants with an introduction to the IEMSR registry, and to provide a general introduction to characteristics of and potential use cases for schema registries in the more general sense of the term.

\section{References}

1. Johnston, P.: What are your terms? Ariadne 53 (2005)

2. Greening, O.: IEMSR Marketing Plan (2006), http: / /www.ukoln.ac.uk/projects/iemsr/phase3 / IEMSR-marketing-plan.pdf

3. The Information Environment Metadata Schema Registry project, http: / /www. ukoln.ac.uk/projects/iemsr/

4. The NSDL metadata registry, http://metadataregistry.org/ 